\title{
ON THE PERIODIC BOUNDARY VALUE PROBLEM FOR IMPULSIVE PARABOLIC EQUATIONS
}

\author{
BY \\ DRUMI BAINOV (Medical University of Sofia, P.O. Box 45, 1504 Sofia, Bulgaria), \\ EMIL MINCHEV (Medical University of Sofia, P.O. Box 45, 1504 Sofia, Bulgaria), \\ AND
}

IKECHUKWU E. OKOROAFOR (Department of Mathematics, University of Port Harcourt, P.M.B. 5323, Port Harcourt, Nigeria)

\begin{abstract}
This paper deals with the periodic boundary value problem for impulsive parabolic equations. A comparison result for impulsive differential inequalities is obtained. This result is applied to get a uniqueness criterion for the solutions of impulsive parabolic equations.
\end{abstract}

1. Introduction. The theory of impulsive partial differential equations is a new branch of the theory of partial differential equations (PDE). Its start was made by the pioneer paper of L. Erbe, H. Freedman, X. Liu, and J. Wu [5]. They showed that impulsive parabolic equations provide a natural framework for mathematical simulations of many processes and phenomena in population dynamics. After the contribution of L. Erbe et al., the theory of impulsive PDEs underwent a rapid development in various directions. We shall mention some of them: quenching phenomena [3], [4], semigroup approaches [6], numerical analyses [1], [2], applications in quantum mechanics [7], etc.

The present paper deals with a periodic boundary value problem for impulsive parabolic equations. Impulsive differential inequalities generated by this problem are considered and uniqueness criteria are obtained. The results of this paper will be actively used as an auxiliary apparatus in the theory of impulsive PDEs.

2. Preliminary notes. Suppose that $\Omega \subset \mathbb{R}^{n}$ is a bounded domain with a boundary $\partial \Omega$ and $\bar{\Omega}=\Omega \cup \partial \Omega$. Let $a_{0}>0$ and let

$$
\begin{aligned}
E & =\left\{(t, x) \in \mathbb{R}^{1+n}: t \in\left[0, a_{0}\right], x \in \Omega\right\}, \\
E^{*} & =\left\{(t, x) \in \mathbb{R}^{1+n}: t \in\left[0, a_{0}\right], x \in \bar{\Omega}\right\} .
\end{aligned}
$$

Received June 13, 1997.

1991 Mathematics Subject Classification. Primary 35R99.

Key words and phrases. Periodic boundary value problem, impulsive parabolic equations.

(C)1999 Brown University 
Suppose that $0<t_{1}<t_{2}<\ldots<t_{k}<a_{0}$ are given numbers and $t_{0}=0, t_{k+1}=a_{0}$.

Define $J_{i m p}=\left\{t_{p}\right\}_{p=1}^{k}, E_{i m p}=\left\{(t, x) \in E: t \in J_{i m p}\right\}, E_{i m p}^{*}=\left\{(t, x) \in E^{*}: t \in\right.$ $\left.J_{i m p}\right\}$.

Let $C_{i m p}\left[E^{*}, \mathbb{R}\right]$ be the class of all functions $z: E^{*} \rightarrow \mathbb{R}$ such that:

(i) the restriction of $z$ to the set $E^{*} \backslash E_{i m p}^{*}$ is a continuous function;

(ii) for each $(t, x) \in E_{i m p}^{*}$ there exist the limits

$$
\lim _{\substack{(s, y) \rightarrow(t, x) \\ s<t}} z(s, y)=z\left(t^{-}, x\right), \quad \lim _{\substack{(s, y) \rightarrow(t, x) \\ s>t}} z(s, y)=z\left(t^{+}, x\right),
$$

and $z(t, x)=z\left(t^{+}, x\right)$.

For a function $z \in C_{i m p}\left[E^{*}, \mathbb{R}\right]$ and $(t, x) \in E_{i m p}^{*}$ we define

$$
\Delta z(t, x)=z(t, x)-z\left(t^{-}, x\right) \text {. }
$$

Let $M[n]$ be the class of all matrices $\gamma=\left[\gamma_{i j}\right]_{1 \leq i, j \leq n}$, where $\gamma_{i j} \in \mathbb{R}$ and $\gamma_{i j}=\gamma_{j i}$.

Suppose that $f:\left(E \backslash E_{i m p}\right) \times \mathbb{R} \times \mathbb{R}^{n} \times M[n] \rightarrow \mathbb{R}, g: E_{i m p}^{*} \times \mathbb{R} \rightarrow \mathbb{R}, \varphi:\left[0, a_{0}\right] \times \partial \Omega \rightarrow$ $\mathbb{R}$ are given functions.

A function $z \in C_{i m p}\left[E^{*}, \mathbb{R}\right]$ will be called a function of class $C_{i m p}^{1,2}\left[E^{*}, \mathbb{R}\right]$ if $z$ possesses continuous partial derivatives $z_{t}(t, x), z_{x}(t, x)$, and $z_{x x}(t, x)$ for $(t, x) \in E \backslash E_{i m p}$, where

$$
z_{x}=\left(z_{x_{1}}, \ldots, z_{x_{n}}\right), \quad z_{x x}=\left[z_{x_{i} x_{j}}\right]_{1 \leq i, j \leq n} .
$$

A function $f:\left(E \backslash E_{i m p}\right) \times \mathbb{R} \times \mathbb{R}^{n} \times M[n] \rightarrow \mathbb{R}$ is said to be elliptic with respect to $z \in C_{i m p}^{1,2}\left[E^{*}, \mathbb{R}\right]$ in $E \backslash E_{i m p}$ if for $(t, x) \in E \backslash E_{i m p}$ and for any $\gamma, s \in M[n]$ such that

$$
\sum_{i, j=1}^{n}\left(\gamma_{i j}-s_{i j}\right) \lambda_{i} \lambda_{j} \leq 0, \quad \lambda=\left(\lambda_{1}, \ldots, \lambda_{n}\right) \in \mathbb{R}^{n},
$$

we have

$$
f\left(t, x, z(t, x), z_{x}(t, x), \gamma\right) \leq f\left(t, x, z(t, x), z_{x}(t, x), s\right) .
$$

We consider the periodic boundary value problem (PBVP):

$$
\begin{aligned}
& z_{t}(t, x)=f\left(t, x, z(t, x), z_{x}(t, x), z_{x x}(t, x)\right), \quad(t, x) \in E \backslash E_{i m p}, \\
& z(0, x)=z\left(a_{0}, x\right), \quad x \in \bar{\Omega}, \\
& z(t, x)=\varphi(t, x), \quad(t, x) \in\left[0, a_{0}\right] \times \partial \Omega, \\
& \Delta z(t, x)=g(t, x, z(t, x)), \quad(t, x) \in E_{i m p}^{*} .
\end{aligned}
$$

Definition 1. The function $z: E^{*} \rightarrow \mathbb{R}$ is a solution of the PBVP (1)-(4) if:

(i) $z \in C_{i m p}^{1,2}\left[E^{*}, \mathbb{R}\right]$ and $z$ satisfies Eq. (1) on $E \backslash E_{i m p}$;

(ii) $z$ satisfies (2), (3) and (4).

The solution $z(t, x)$ of $\operatorname{PBVP}(1)-(4)$ is called parabolic if for every $(t, x) \in E \backslash E_{\text {imp }}$ all the functions $f(t, x, z, p, q)$ are elliptic with respect to $z(t, x)$. 
3. Main results. Now we prove a theorem on the differential inequalities generated by PBVP (1)-(4).

THEOREM 1. Let the following conditions hold:

1. $u, v \in C_{i m p}^{1.2}\left[E^{*}, \mathbb{R}\right]$ and the function $f$ is elliptic with respect to $u$ in $E \backslash E_{i m p}$.

2. The differential inequalities

$$
\begin{aligned}
& u_{t}(t, x) \leq f\left(t, x, u(t, x), u_{x}(t, x), u_{x x}(t, x)\right), \\
& v_{t}(t, x) \geq f\left(t, x, v(t, x), v_{x}(t, x), v_{x x}(t, x)\right)
\end{aligned}
$$

hold on $E \backslash E_{i m p}$ and

$$
\begin{aligned}
& u(0, x) \leq u\left(a_{0}, x\right), \quad v(0, x) \geq v\left(a_{0}, x\right), \quad x \in \bar{\Omega}, \\
& u(t, x) \leq v(t, x), \quad(t, x) \in\left[0, a_{0}\right] \times \partial \Omega .
\end{aligned}
$$

3. For each $(t, x) \in E_{i m p}^{*}$ we have

$$
\begin{aligned}
& \Delta u(t, x) \leq g(t, x, u(t, x)), \\
& \Delta v(t, x) \geq g(t, x, v(t, x)) .
\end{aligned}
$$

4. For each $(t, x) \in E \backslash E_{i m p}$ the function $f(t, x, \cdot, p, q): \mathbb{R} \rightarrow \mathbb{R}$ is strictly decreasing on $\mathbb{R}$.

5. For each $(t, x) \in E_{i m p}$ the function $g(t, x, \cdot): \mathbb{R} \rightarrow \mathbb{R}$ is strictly decreasing on $\mathbb{R}$. Then we have

$$
u(t, x) \leq v(t, x) \quad \text { on } E^{*} .
$$

Proof. Let $w(t, x)=u(t, x)-v(t, x)$ for $(t, x) \in E^{*}$ and

$$
\varepsilon=\sup \left\{w(t, x):(t, x) \in E^{*}\right\}
$$

Suppose (8) is not true. Then $\varepsilon>0$ and there are two cases to be distinguished:

Case 1. There exists $(\tilde{t}, \tilde{x}) \in E^{*}, \tilde{x}=\left(\tilde{x}_{1}, \ldots, \tilde{x}_{n}\right)$, such that

$$
w(\tilde{t}, \tilde{x})=\varepsilon, \quad w(t, x) \leq \varepsilon \quad \text { on } E^{*} .
$$

It follows from (6) that we can take $\tilde{t}>0$ and from (7) that $\tilde{x} \in \Omega$. have

(1a). Suppose that $(\tilde{t}, \tilde{x}) \in E \backslash E_{i m p}$. Since $u-v$ attains its maximum at $(\tilde{t}, \tilde{x})$ we

$$
u_{t}(\tilde{t}, \tilde{x}) \geq v_{t}(\tilde{t}, \tilde{x}), \quad u_{x}(\tilde{t}, \tilde{x})=v_{x}(\tilde{t}, \tilde{x})
$$

and

$$
\sum_{i, j=1}^{n}\left(u_{x_{i} x_{j}}(\tilde{t}, \tilde{x})-v_{x_{i} x_{j}}(\tilde{t}, \tilde{x})\right) \lambda_{i} \lambda_{j} \leq 0
$$

for $\lambda=\left(\lambda_{1}, \ldots, \lambda_{n}\right) \in \mathbb{R}^{n}$. 
In view of (5) and condition 4 of the theorem it follows that

$$
\begin{aligned}
0 \geq & v_{t}(\tilde{t}, \tilde{x})-u_{t}(\tilde{t}, \tilde{x}) \geq f\left(\tilde{t}, \tilde{x}, v(\tilde{t}, \tilde{x}), v_{x}(\tilde{t}, \tilde{x}), v_{x x}(\tilde{t}, \tilde{x})\right) \\
& \quad-f\left(\tilde{t}, \tilde{x}, u(\tilde{t}, \tilde{x}), u_{x}(\tilde{t}, \tilde{x}), u_{x x}(\tilde{t}, \tilde{x})\right) \\
\geq & f\left(\tilde{t}, \tilde{x}, v(\tilde{t}, \tilde{x}), v_{x}(\tilde{t}, \tilde{x}), v_{x x}(\tilde{t}, \tilde{x})\right)-f\left(\tilde{t}, \tilde{x}, v(\tilde{t}, \tilde{x})+\varepsilon, v_{x}(\tilde{t}, \tilde{x}), v_{x x}(\tilde{t}, \tilde{x})\right)>0,
\end{aligned}
$$

which is a contradiction.

(1b). $(\tilde{t}, \tilde{x}) \in E_{i m p}$. Then we have

$$
u\left(\tilde{t}^{-}, \tilde{x}\right) \leq v\left(\tilde{t}^{-}, \tilde{x}\right)+\varepsilon .
$$

It follows that

$$
u(\tilde{t}, \tilde{x})-v(\tilde{t}, \tilde{x}) \leq u\left(\tilde{t}^{-}, \tilde{x}\right)+g(\tilde{t}, \tilde{x}, u(\tilde{t}, \tilde{x}))-[v(\tilde{t}-\tilde{x})+g(\tilde{t}, \tilde{x}, v(\tilde{t}, \tilde{x}))]<\varepsilon,
$$

which is a contradiction with (9).

Case 2. Suppose that $w(t, x)<\varepsilon$ for $(t, x) \in E^{*}$. Then there is $(\tilde{t}, \tilde{x}) \in E$ such that

$$
\lim _{(t, x) \rightarrow\left(\tilde{t}^{-}, \tilde{x}\right)} w(t, x)=\varepsilon
$$

and there is $j, 1 \leq j \leq k$ such that $\tilde{t}=t_{j}$. Let $\tilde{\eta}:\left[t_{j-1}, t_{j}\right] \rightarrow \mathbb{R}$ be defined by

$$
\tilde{\eta}(t)=\max \{w(t, x): x \in \bar{\Omega}\}
$$

for $t \in\left[t_{j-1}, t_{j}\right)$ and $\tilde{\eta}\left(t_{j}\right)=\varepsilon$. Thus, $\tilde{\eta}$ is a continuous function. Since $\tilde{\eta}(t)<\varepsilon$ for $t \in\left[t_{j-1}, t_{j}\right)$, there is a sequence $\left\{\tilde{t}_{i}\right\}$ such that

$$
\tilde{t}_{i} \in\left[t_{j-1}, t_{j}\right), \quad \tilde{t}_{i}<\tilde{t}_{i+1}, \quad \lim _{i \rightarrow \infty} \tilde{t}_{i}=t_{j}=\tilde{t}
$$

and

$$
D_{-} \tilde{\eta}\left(\tilde{t}_{i}\right) \geq 0 \quad \text { for } \quad i=1,2, \ldots,
$$

where $D_{-}$is the Dini derivative. For each $i$ there exists $\tilde{x}^{(i)} \in \bar{\Omega}$ such that

$$
\tilde{\eta}\left(\tilde{t}_{i}\right)=w\left(\tilde{t}_{i}, \tilde{x}^{(i)}\right) \text {. }
$$

It follows from (7) and (10) that there exists a natural number $N$ such that for $i \geq N$, $\left(\tilde{t}_{i}, \tilde{x}^{(i)}\right) \in E$ and $w\left(\tilde{t}_{i}, \tilde{x}^{(i)}\right)>0$. Then we have

$$
\begin{aligned}
0 \leq & D_{-} \tilde{\eta}\left(\tilde{t}_{i}\right) \leq w_{t}\left(\tilde{t}_{i}, \tilde{x}^{(i)}\right)=u_{t}\left(\tilde{t}_{i}, \tilde{x}^{(i)}\right)-v_{t}\left(\tilde{t}_{i}, \tilde{x}^{(i)}\right) \\
\leq & f\left(\tilde{t}_{i}, \tilde{x}^{(i)}, u\left(\tilde{t}_{i}, \tilde{x}^{(i)}\right), u_{x}\left(\tilde{t}_{i}, \tilde{x}^{(i)}\right), u_{x x}\left(\tilde{t}_{i}, \tilde{x}^{(i)}\right)\right) \\
& \quad-f\left(\tilde{t}_{i}, \tilde{x}^{(i)}, v\left(\tilde{t}_{i}, \tilde{x}^{(i)}\right), v_{x}\left(\tilde{t}_{i}, \tilde{x}^{(i)}\right), v_{x x}\left(\tilde{t}_{i}, \tilde{x}^{(i)}\right)\right)<0,
\end{aligned}
$$

since

$$
u_{x}\left(\tilde{t}_{i}, \tilde{x}^{(i)}\right)=v_{x}\left(\tilde{t}_{i}, \tilde{x}^{(i)}\right)
$$

and

$$
\sum_{i, j=1}^{n}\left(u_{x_{i} x_{j}}\left(\tilde{t}_{i}, \tilde{x}^{(i)}\right)-v_{x_{i} x_{j}}\left(\tilde{t}_{i}, \tilde{x}^{(i)}\right)\right) \lambda_{i} \lambda_{j} \leq 0
$$

for $\lambda=\left(\lambda_{1}, \ldots, \lambda_{n}\right) \in \mathbb{R}^{n}$. Thus we have obtained a contradiction. 
Therefore

$$
u(t, x) \leq v(t, x) \quad \text { on } E^{*} .
$$

We apply Theorem 1 to obtain a uniqueness result for PBVP (1)-(4).

THEOREM 2. Let the following conditions hold:

1. for each $(t, x) \in E \backslash E_{i m p}$ the function $f(t, x, \cdot, p, q): \mathbb{R} \rightarrow \mathbb{R}$ is strictly decreasing on $\mathbb{R}$;

2. for each $(t, x) \in E_{i m p}$ the function $g(t, x, \cdot): \mathbb{R} \rightarrow \mathbb{R}$ is strictly decreasing on $\mathbb{R}$.

Then the PBVP (1)-(4) admits at most one parabolic solution.

Proof. Let $u_{1}, u_{2} \in C_{i m p}^{1,2}\left[E^{*}, \mathbb{R}\right]$ be two distinct parabolic solutions of the PBVP (1)-(4). Employing Theorem 1 we can prove that:

$$
\begin{aligned}
& \text { (i) } u_{1} \leq u_{2} \quad \text { on } E^{*} \text {, } \\
& \text { (ii) } u_{1} \geq u_{2} \text { on } E^{*} \text {, }
\end{aligned}
$$

which imply the statement of the theorem.

Acknowledgments. The present investigation was partially supported by the Bulgarian Ministry of Education, Science and Technologies under Grant MM-702.

\section{REFERENCES}

[1] D. Bainov, Z. Kamont, and E. Minchev, Difference methods for impulsive differential-functional equations, Applied Numerical Mathematics 16, 401-416 (1995)

[2] D. Bainov, Z. Kamont, and E. Minchev, The finite difference method for first order impulsive partial differential-functional equations, Computing 55, No. 3, 237-253 (1995)

[3] C. Y. Chan and L. Ke, Remarks on impulsive quenching problems, Proceedings of Dynamic Systems and Applications 1, 59-62 (1994)

[4] C. Y. Chan, L. Ke, and A. Vatsala, Impulsive quenching for reaction-diffusion equations, Nonlinear Analysis, Theory, Methods and Applications 22, No. 11, 1323-1328 (1994)

[5] L. H. Erbe, H. I. Freedman, X. Z. Liu, and J. H. Wu, Comparison principles for impulsive parabolic equations with applications to models of single species growth, J. Austral. Math. Soc., Ser. B, 32, 382-400 (1991)

[6] V. Gupta, Parabolic Equations with Impulse Effect: a Semigroup Approach, Ph.D. Thesis, Kanpur, India, 1994

[7] G. Petrov, Impulsive moving mirror model in a Schrödinger picture with impulse effect in a Banach space, Communications of the Joint Institute for Nuclear Research, Dubna, Russia, preprint E292-272, 1992 